\title{
Desastres naturais: \\ convivência com o risco
}

\author{
SAMIA NASCIMENTO SULAIMAN ${ }^{I}$ \\ e ANTONIO ALEDO ${ }^{I I}$
}

\section{Introdução}

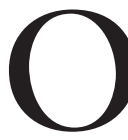

S PROGNÓSTICOS negativos sobre alterações no clima e os impactos dos eventos naturais cada vez mais recorrentes e catastróficos têm ganhado repercussão nas agendas de governos e instituições internacionais, bem como nos meios de comunicação e no cotidiano da sociedade contemporânea. As estratégias de ação, que se centravam na resposta a esses impactos, têm-se direcionado ao estudo, planejamento e intervenção sobre situações e contextos de risco, antes da materialização do impacto. Nesse contexto, enfoca-se a relevância de se construir uma cultura de segurança para mitigação e prevenção sobre os riscos de desastres.

Os estudos sobre riscos de desastres vêm ganhando diferentes abordagens e apoiando diferentes medidas de gestão, que podem ser tipificadas em paradigmas que coexistem entre discursos e práticas (Warner et al., 2002; Smith; Petley, 2009). Para Juneja (2009, p.2), há uma lacuna conceitual, por um lado, pela aceitação entre os claim makers do discurso sobre os benefícios de uma abordagem integrada que enfatiza a vulnerabilidade e, por outro, pela hegemonia da visão dominante na prática de gestão de risco que favorece soluções tecnológicas, de cima para baixo (top-down), minimizando a análise e tratamento de causas sociais e políticas dos riscos.

Frente a isso, Funtowicz e Ravetz (2000) publicizaram, em 1993, um novo paradigma de ciência para atuar frente às doenças do sistema industrial global do qual ela própria forma base: a ciência pós-normal. A proposta da ciência pós-normal (Funtowicz; Ravetz, 2000) busca superar a ciência normal (Kuhn, 2005), cartesiana e positivista na resolução de problemas, baseada na segurança e controle sobre o mundo natural. A inserção das noções de probabilidade, incerteza e risco vão permitir a emersão desse novo paradigma científico que se apoia na Qualidade, e não na Verdade, do conhecimento científico constituído e legitimado por uma "comunidade ampliada de pares" (Funtowicz; De Marchi, 2003 ) para além da comunidade científica. Coloca, ainda, o papel da ciência na “sensibilização" (Wesselink; Hoppe, 2013) de uma política de governança dos problemas ambientais, a qual aponta a abertura da gestão pública à participação da sociedade, num processo de governança ambiental, baseada na corresponsabilização. 
Para a integração de atores sociais, é necessário mediação. No campo da prevenção de desastres, no entanto, os processos de informação, comunicação e educação têm-se apoiado na "convivência com o risco". Em prol de uma cultura de segurança, são disseminados conhecimentos técnico-científicos que permitam entender os fenômenos naturais e identificar áreas de risco, ao mesmo tempo que são indicadas medidas de prevenção e resposta para reduzir os impactos de um evento extremo. Apesar da relevância desse saber, o diagnóstico e o mapeamento das situações e contextos de risco enfocam o estado presente, verificável, e ocultam os processos sócio-históricos de formação de áreas de risco, bem como a desigualdade dos impactos sociais dos desastres, e os processos educativos perdem o potencial de formação crítica e participativa da sociedade para uma gestão compartilhada (Sulaiman, 2014).

Este artigo apresenta uma revisão bibliográfica sobre o pensamento hegemônico que limita a gestão de riscos de desastres, bem como os processos de comunicação e educação, a conhecimentos e medidas técnico-científicas, constituindo, de um lado, a tomada de decisão de cima para baixo e, de outro, a desvalorização dos conhecimentos e da participação da sociedade. Essa limitação é explicada a partir do conceito de "inquestionabilidade do risco" (Aledo; Sulaiman, 2014, 2015), pelo qual se identifica uma incapacidade cultural e cognitiva de especialistas, científicos e tomadores de decisão de identificar e atuar sobre as causas profundas da produção de risco e, portanto, questionar o modelo de sociedade contemporânea. $\mathrm{O}$ conceito é analisado nos conteúdos e nas práticas educativas que, ao se ensinar como identificar situações de risco e o que fazer, não explicitam os processos sócio-históricos de formação de áreas de risco nem fortalecem a perspectiva crítica e mobilizadora da educação para prevenção de desastres.

O texto finaliza-se com a análise dos princípios da ciência pós-normal, que rompe com a hegemonia do paradigma de ciência, política e sociedade modernas, ao abarcar um conceito sistêmico que inclui e legitima uma pluralidade de perspectivas de respostas, defendendo não a Verdade, mas a Qualidade como princípio organizador. Para isso, considera risco, incerteza e probabilidade na construção do conhecimento científico a partir da ampliação tanto dos "fatos" relevantes quanto do número e tipo de atores, individuais e/ou coletivos, legitimados para intervir na definição dos problemas tanto como na seleção e implementação das políticas conectadas (Funtowicz; De Marchi, 2003).

\section{O pensamento hegemônico e a inquestionabilidade do risco}

$\mathrm{Na}$ gestão e planejamento frente aos riscos de desastres há o predomínio de políticas de controle da natureza, alicerçadas em avançada capacidade geofísica, geotécnica e administrativa: frente a eventos pluviométricos extremos como secas, represas e sistemas de irrigação; como inundações, sistemas de monitoramento, prognóstico e alarme (Burton et al., 1978, p.219). O pressuposto dessa visão e ação está na compreensão de que os desastres naturais estão na relação 
proporcional entre determinadas forças da natureza e o nível de avanço técnico e institucional para fazer frente a elas (Hewitt, 1983).

A preponderância do controle sobre a natureza como forma de garantir segurança, especialmente nas áreas urbanas, debilita a gestão do risco que não considera as desigualdades dos impactos sociais de um desastre. Os resultados da passagem do furacão Katrina, em 2005, em Nova Orleans, no sul dos Estados Unidos, são um exemplo. A ocorrência de um furacão tropical já constava entre os gestores de emergência; assim como as intervenções estruturais já controlavam as inundações naturais (Nova Orleans localiza-se entre três corpos d'água: Lago Pontchartrain, Golfo do México e Rio Mississippi). No entanto, o uso e a ocupação do solo produziram uma geografia social plena de vulnerabilidades, relacionadas a um antigo passado de segregação racial e social: a população negra ocupou áreas menos valorizadas e possuía limitações de acesso a emprego, educação de qualidade e perspectivas de futuro (Cutter, 2006).

As consequências do desastre, portanto, não estiveram apenas relacionadas com falhas no sistema de gestão de emergência, que podem ser rapidamente identificadas e corrigidas, mas sim com a debilidade dos sistemas de apoio social para os empobrecidos estadunidenses, "invisibilizados" nas cidades do interior do país, como Nova Orleans, "The City that Care Forgot" (op. cit.). A gestão da vulnerabilidade demanda tempo, recurso e vontade política para corrigir as desigualdades e assimetrias produzidas e perpetuadas, no caso dos Estados Unidos, por mais de dois séculos e ainda presentes.

A produção da vulnerabilidade social está imbricada no atual sistema socioeconômico que se configurou sobre o mito do desenvolvimento (Furtado, 1974). Com base no crescimento contínuo erigido sobre o controle da natureza; na ciência e tecnologia capacitando especialistas para o controle de sistemas cada vez mais complexos; e no mercado como o agente capaz de gerir de forma sustentável as relações entre sociedade, economia e meio ambiente, a sociedade contemporânea erigiu três imperativos, universais e inquestionáveis, que, contraditoriamente, são os produtores de risco e de insustentabilidade do próprio sistema (Blühdorn, 2000; Blühdorn; Welsh, 2007).

O crescimento econômico resulta em taxas de produção de entropia que geram a degradação dos serviços ecossistêmicos que, por sua vez, provocam um aumento da vulnerabilidade das populações humanas dependentes desses serviços. A ciência e a tecnologia escaparam ao controle social, gerando riscos que distribuem de forma desigual, ao afetar em maior medida populações situadas em classes inferiores da estrutura socioeconômica. Por último, o mercado também intensifica a desigualdade, e a concentração de riquezas em elites globais provém da espoliação de recursos dos grupos humanos mais indefesos. A isso acresce-se a produção de contextos de vulnerabilidade.

Diante desse cenário, são relevantes os trabalhos de Wisner, Blaikie, Cannon e Davis (2004) que abordam as causas dos desastres naturais em diferentes 
escalas, tempos e espaços como um processo sócio-histórico de desdobramentos e inter-relações a partir da "progressão da vulnerabilidade", Segundo os autores, há três escalas de análise da vulnerabilidade: "causas profundas", "pressões dinâmicas" e "condições inseguras". As "causas profundas" estão distantes do problema concreto, na origem da vulnerabilidade, e relacionam-se com a distribuição de poder e o modelo de organização social. As "pressões dinâmicas" são processos e atividades que decorrem das "causas profundas", e podem ser exemplificadas com crescimento populacional, políticas assistencialistas, falta de participação social, degradação ambiental, ocupação ilegal/irregular. As "condições inseguras" são as formas específicas que materializam a vulnerabilidade no tempo e espaço, como habitações precárias, falta de rede de drenagem, analfabetismo, desemprego etc. Essa abordagem permite entender que as condições social e historicamente construídas fazem que eventos naturais, mesmo de baixa magnitude e intensidade, se tornem catástrofes. Não considerar a vulnerabilidade ou ainda considerar parcialmente suas causas significa ignorar as bases de desenvolvimento da sociedade moderna.

Apesar da relevância do enfoque da vulnerabilidade, e de seus diferentes níveis causais, permanecem resistências para incorporá-la na gestão de riscos de desastres. No campo da gestão de riscos de desastres, essa configuração de pensamento e ação resulta no que chamamos de "inquestionabilidade do risco" (Aledo; Sulaiman, 2015). Há um pensar e agir sobre o risco existente, instalado a partir do paradigma hegemônico da ciência "normal", devido a uma incapacidade cultural e política de enfrentar as causas produtoras do risco, as quais residem no modelo socioeconômico e científico da sociedade contemporânea.

\section{Entre saber para conviver e para mudar}

A ação educativa, como toda ação humana, responde às crenças e exigências de seu tempo. Numa perspectiva intercultural da educação e com o desenvolvimento tecnológico dos meios de comunicação, reconhece-se a multiplicidade de meios e espaços de aprendizagem, para além da escola. Além disso, a crise das certezas científicas e as metas da sustentabilidade têm colocado novos dilemas e desafios à educação contemporânea. A discussão sobre os rumos da humanidade em prol do desenvolvimento sustentável e da resiliência é uma oportunidade para se pensar a relação entre educação e sociedade, assim como seu papel na prevenção de desastres naturais.

A primeira questão que um processo educativo crítico para prevenção de desastres poderia se fazer é sobre conflitos e polêmicas em torno da operacionalização dos conceitos de desenvolvimento sustentável e resiliência. O termo "desenvolvimento sustentável" foi cunhado para conciliar interesses econômicos e socioambientais (CMMAD, 1988). O termo oscila entre um avançado sentido socioambiental de desenvolvimento, questionando as bases ideológicas de produção capitalista da sociedade moderna, e uma estratégia conservadora de manutenção do crescimento econômico com uma variável ecológica (Lima, 
2003, p.105; Gadotti, 2008, p.61). No caso da resiliência, o termo, extrapolado do campo da Física, tem sua aplicação estendida da Ecologia à Inovação Social e tem tornado fundamento para a adaptação da gestão urbana aos desastres naturais e às mudanças climáticas, referindo-se à capacidade de resistência da sociedade frente a processos/eventos naturais extremos (Timmerman, 1981). Em vez de se superar o paradigma da sociedade capitalista moderna, altamente consumidora de hidrocarburetos e produtora de exclusão/segregação socioespacial e de risco, tornar-se resiliente tem envolvido instrumentalização, formação e capacitação de grupos humanos, comunidades, cidades vulneráveis a desastres naturais, para a convivência com o risco, perpetuando uma perspectiva conservadora de aceitação do que está posto, inquestionável, negligenciando a produção social do risco e os impactos desigualmente distribuídos. Nesse contexto, o desenvolvimento sustentável e a resiliência tornam-se uma adaptação às "externalidades" do desenvolvimento da sociedade moderna e não uma transformação frente às causas produtoras do risco, mantendo a "inquestionabilidade do risco".

A educação direcionada à redução dos riscos de desastres tem abordado o que são riscos de desastres naturais e como atuar para enfrentá-los, por meio de processos de conscientização pública e capacitação de modo a modificar a percepção de risco e motivar ações de autoproteção. Incialmente, com o protagonismo das instituições de ajuda humanitária e de ação militar, enfocou a disseminação dos tipos de risco e ações de preparação e resposta (Lidstone, 1999; Petal et al., 2008). Os conteúdos educativos, nesse contexto, colocaram a prevenção como sinônimo de preparação para uma resposta adequada em situação de crise, perspectiva que perpetua. Sob o conceito de educação para a preparação frente aos desastres naturais (education for natural disaster preparedness), a Década das Nações Unidas da educação para o desenvolvimento sustentável 2005-2014 (Unesco, 2005, p.55), apontou que:

as crianças que sabem como reagir no caso de terremoto, os líderes comunitários que aprenderam a avisar a sua comunidade a tempo de se protegerem dos riscos e camadas sociais inteiras que foram ensinadas a se preparar para enfrentar desastres naturais contribuem para melhorar as estratégias de atenuação dos efeitos dos desastres. Educação e saber forneceram à sociedade estratégias de autoajuda que diminuem sua vulnerabilidade e melhoram sua vida.

A partir de premissas como "informação gera proteção" e "conhecer para prevenir", a educação para a redução de riscos de desastres valoriza o conhecimento técnico-científico acumulado sobre desastres e deixa de considerar o conhecimento local, experienciado para esse processo. Há uma gestão da informação e não do conhecimento (De Long et al., 1997) e perde-se o papel transformador da educação, especialmente no caso dos desastres, apontado por $\operatorname{Leff}(2003$, p.58):

a educação deve preparar as novas gerações não somente para aceitar a incerteza (uma educação como preparação ante o desastre ecológico e capacidades 
de respostas para o imprevisto); também deve preparar novas mentalidades capazes de compreender as complexas inter-relações entre os processos objetivos e subjetivos que constituem seu mundo de vida, para gerar habilidades inovadoras para a construção do inédito. Trata-se de uma educação que permite preparar-se para a construção de uma nova racionalidade; não para uma cultura de desesperança e alienação; ao contrário, para um processo de emancipação que permita novas formas de reapropriação do mundo.

Na prática, os processos de aprendizagem buscam desenvolver a percepção do risco "com o intuito de transmutar a simples intuição psicológica que possuem sobre os eventos que possam ocorrer, em um olhar mais técnico e, portanto mais real dos problemas aos quais estão sujeitos, bem como alertá-los sobre como agir em situações de necessidade" (Silva; Macedo, 2007). Por extensão, o "olhar da população" é entendido como "menos real", desconsiderando-se tanto o saber experienciado da população - principalmente dos moradores em área de risco que sabem pela vivência cotidiana com o risco - quanto a participação na construção coletiva e integrada da gestão de risco de desastre. Petal et al. (2008) definem essa questão como "negação de conhecimento" (knowledge denial), uma das faces do processo de opressão para a redução de risco de desastre. Opressão pela não contestação do crescimento ilimitado sob o controle da natureza, pela não contestação da certeza da ciência e tecnologia em explicar e resolver problemas, pela não contestação do poder do mercado na regulação do desenvolvimento material.

A educação para a prevenção de desastres e a construção de uma cultura de segurança apoia-se no "conhecer para prevenir" com foco na disseminação e apreensão de conhecimentos científicos para a convivência com o risco, limitando a construção social e participativa de conhecimentos e soluções que enfrentem as bases sociais de produção de riscos e de impactos socialmente desiguais.

Para a viabilização da mudança social e a reorientação da sociedade em direção à sustentabilidade, Keen et al. (2005) apontam a necessidade de três novas agendas de aprendizagem baseadas na valorização, integração e compartilhamento de saberes. Indicam, portanto, a formação de espaços e estruturas equitativas de aprendizagem entre comunidades, profissionais e governos para resolver conflitos de forma colaborativa e tomar decisões coletivas, orientando os processos de aprendizagem à mudança social. No campo da gestão ambiental, essa abordagem tem sido colocada como Aprendizagem Social.

O conceito de Aprendizagem social (Social Learning) tem um longo percurso histórico com diferentes abordagens e contextos. Enquanto um conceito moderno relativo à gestão ambiental, para além do campo da psicologia behaviorista ou comportamentalista, parte da premissa de que as decisões sociais podem ser melhoradas por meio do aumento do número de oportunidades de práticas de diálogo, para além da obtenção de apenas um consenso de opinião. A integração de stakeholders, de acordo com Kilvington (2007, 2010), permite compor um quadro analítico construído a partir da mediação entre diferentes 
pontos de vista e fontes de informação, que pode ser utilizado para apoiar a decisão coletiva na gestão de recursos naturais. Essa abordagem também tem sido internalizada para a construção do conhecimento científico por meio de um novo paradigma que considera a probabilidade, o risco e a incerteza e, frente a isso, aposta, não na verdade, mas na qualidade do conhecimento científico construído a partir da mediação entre conhecimentos e saberes oriundos de diferentes stakeholders em interlocução.

\section{Uma contraposição à ciência positivista e a integração de saberes}

As ciências aplicadas e seus padrões para resolução de problemas tradicionalmente representam um conhecimento seguro e um controle eficiente sobre o mundo natural. No entanto, a modernidade produziu, ao mesmo tempo, segurança e insegurança. Segundo Beck (1992), o risco, na sociedade pós-industrial ou pós-moderna, torna-se um dos componentes básicos da estruturação da sociedade. Na teoria da "Sociedade de Risco", os riscos, e a perspectiva de probabilidade e incerteza que trazem, são uma construção social contemporânea, resultado do próprio processo de modernização e desenvolvimento científico e são onipresentes para o indivíduo. Segundo Giddens et al. (1997), a origem do risco alicerça-se em duas questões-chave: a "reflexividade", pela qual se evidencia que as próprias verdades científicas produzem incertezas; e a "falta de referentes", pela qual se constata a falta de referência alternativa à ciência cartesiana, racionalista para a construção de conhecimento e proposição de ações e intervenções.

O risco, portanto, coloca em questão o pensamento racionalista-positivista-cartesiano, alicerçado nas leis da Física de teor clássico, que entendia o mundo como uma máquina e analisava suas diferentes partes separadamente. Nos anos 1980, Capra (1982) criticou o reducionismo desse pensamento e a emergência de um novo paradigma, sistêmico, holístico, integrador. Diante da crise ambiental, coloca-se o desafio na construção de uma nova racionalidade, no corpo das ciências naturais e sociais, para "construir um conhecimento capaz de integrar a multicausalidade e as relações de interdependência dos processos da ordem natural e social que determinam, condicionam e afetam as mudanças socioambientais, assim como para construir uma racionalidade produtiva fundada nos princípios do desenvolvimento sustentável" (Leff, 2006, p.239).

A proposta da ciência pós-normal (Funtowicz; Ravetz, 2000) busca superar a ciência normal (Kuhn, 2005), cartesiana e positivista na resolução de problemas, baseada na segurança e controle sobre o mundo natural. A inserção das noções de probabilidade, incerteza e risco vai permitir a emersão desse novo paradigma científico que se apoia na Qualidade, e não na Verdade, do conhecimento científico constituído e legitimado por uma "comunidade ampliada de pares" (Funtowicz; De Marchi, 2003) para além da comunidade científica.

Wesselink e Hoppe (2013) colocam o papel da ciência na "sensibilização" de uma política de governança dos problemas ambientais, a qual aponta a abertura da gestão pública à participação da sociedade, num processo de governança 
ambiental, baseada na corresponsabilização. Para Funtowicz e Ravetz (1997, p.229), enfraquece-se a lógica de "predição científica" e a visão dominante da "explicação científica" em prol de uma abordagem mais pragmática de "previsão de políticas" e "compreensão societária".

Evidencia, assim, a necessidade de se considerar a relação complexa e coevolutiva entre teorias científicas e a compreensão científica das mudanças dos ecossistemas e a evolução das instituições sociais e o imaginário cultural (Norgaard, 1994). É o que Acselrad (2009, p.77) defende: "de um lado apoio à 'ciência popular', promovida por um conjunto de trabalhadores pioneiros que pode ajudar a projetar e a implementar estratégias eficientes em termos de custo para monitorar problemas identificados por grupos comunitários, e de outro, uma sofisticada ciência de interesse público". Funtowicz e De Marchi (2003, p.84) enxergam essa perspectiva dentro da "pesquisa científica para a sustentabilidade":

por um lado, é necessário ampliar a categoria dos "fatos" relevantes para a análise e a gerência dos problemas ambientais. Por outro lado, é obrigatório ampliar o número e tipo de atores, tanto individuais como coletivos, legitimados para intervir na definição dos problemas tanto como na seleção e implementação das políticas conectadas. Em outras palavras, a maneira adequada de conduzir um processo de decisão influi dramaticamente sobre seus resultados. Além dos "fatos" ampliados devemos reconhecer as comunidades de pares ampliadas.

A ciência pós-normal demanda um modelo integrado e colaborativo de produção e validação do conhecimento científico que considera os diferentes tipos de incerteza e a coexistência de uma variedade de perspectivas legítimas. Para Ravetz e De Marchi (1999), há três questões fundamentais nesse novo paradigma científico: a avaliação dos riscos e administração das incertezas; os procedimentos e a participação; e os problemas de governança. A relação entre a limitação do conhecimento científico e a potencialidade da inclusão de saberes e atores sociais para a gestão dos desastres naturais, numa perspectiva compartilhada de governança, são elementos que caracterizam o paradigma da ciência pós-normal na gestão dos riscos.

Após vinte anos da publicação do primeiro trabalho de Funtowicz e Ravetz (2000), Wesselink e Hoppe (2013) reconhecem o êxito da ciência pós-normal como um "conceito sensibilizador". Os autores evidenciaram exemplos concretos de aplicação da ciência pós-normal, utilizada como um método de explicitação das incertezas; como também concluíram que a ciência pós-normal aponta uma relação produtiva entre estudos de ciência e tecnologia e de ciência política. No entanto, na narrativa dominante das políticas, o aumento da incerteza desencadeia pressões para a permanência do status quo (Roe, 1994, p.5), na contramão do que predicam Funtowicz e Ravetz.

\section{Conclusões}

Um feito a considerar na análise do risco ante desastres reside na sua desigual distribuição. Por mais que, frente a riscos sistêmicos, todos os grupos 
sociais possam ser afetados; os chamados riscos acumulativos, ou riscos de desastres socionaturais (unnatural disasters), têm uma maior probabilidade de afetar a determinados grupos socioeconômicos ou sociodemográficos. Aqueles que detêm o poder e que controlam o processo de decisões ante o risco possuem um conjunto de capitais que lhes permite deslocar o risco no tempo (para futuras gerações), no espaço (para outras regiões) e/ou para outros grupos sociais. Dessa maneira, enquanto essas elites conseguem monopolizar os benefícios originados por suas decisões, o risco e seus impactos efetivos são socializados, especialmente entre os mais vulneráveis. Por exemplo, as elites controlam o planejamento urbanístico das cidades. Mediante os processos de colonização urbana, apropriam-se dos territórios mais seguros e deslocam os grupos mais desfavorecidos para as zonas de maior risco ambiental. Assim, enquanto as elites geram espaços de vulnerabilidade socioespacial, alimentam seu enriquecimento com processos especulativos em torno do negócio urbanístico.

A ciência normal e sua aplicação técnica favorece esses processos de desigual distribuição de riscos ao colocar os problemas ambientais como assuntos de gestão e não problematizar com uma reflexão sobre os processos sócio-históricos de produção de risco. É necessário, portanto, novos modelos de gestão de risco e novos modelos educativos emancipadores que questionem essa desigual distribuição do risco e as causas sociais produtoras de vulnerabilidade. Essa proposta nos leva a uma nova ciência radicalmente distinta tanto em sua axiologia - orientada a valores, não somente a fins - e epistemológica - remodeladora da relação entre investigador e investigado.

A proposta de ampliar a comunidade de pares nos processos decisórios ligados a dinâmicas deliberativas - expondo-se, de forma aberta, transparente e democrática, todos os argumentos - permitiria radicalizar a proposta da ciência pós-normal. Entendemos essa radicalização em três âmbitos: primeiro, como um exercício de democracia radical em que se superassem as debilidades da democracia parlamentária e se levasse a democracia ao terreno da discussão popular e cotidiana dos problemas e ameaças ambientais; segundo, como um exercício indagatório e deliberativo de busca das causas últimas geradoras do risco e da vulnerabilidade, desvelando as causas sócio-históricas produtoras e buscando democraticamente a melhor solução; por último, como uma nova forma de educação popular, de aprendizagem social, a partir da participação e interação de todos os grupos sociais interessados (stakeholders). Essa aprendizagem social, produto de processos deliberativos e participativos orientados à resolução dos problemas que afetam aos mais vulneráveis, abriria o caminho para uma educação para prevenção de natureza emancipadora e crítica que permitisse o questionamento coletivo das causas sociais produtoras de risco. 
Referências

ACSELRAD, H. (Org.) Duração das cidades: sustentabilidade e risco nas políticas urbanas. Rio de Janeiro: Lamparina, 2009.

ALEDO, A.; SULAIMAN, S. La incuestionabilidad del riesgo. Revista Ambiente e Sociedade [online], v.17, n.4, p.9-16, 2014.

La incuestionabilidad del riesgo: vulnerabilidade social y riesgo sísmico en municípios turísticos. Cuadernos de Turismo, n.36, p.17-37, 2015.

BECK, U. Risk society: towards a new modernity. Los Angeles; London: Sage, 1992.

BLÜHDORN, I. Post-ecologist politics: social theory and the abdication of the ecologist paradigm. London: Routledge, 2000.

BLÜHDORN, I.; WELSH, I. Eco-politics beyond the Paradigm of Sustainability. Environmental Politics, v.16, n.2, p.185-205, 2007. Disponível em: <http://goo.gl/ ShTu9n>, Acesso em: $1^{\circ}$ ago. 2016.

BUCKLE, P. Prospects for public sector disaster management in the 1990s: an indication of current issues with particular reference to Victoria, Australia. International Journal of Mass Emergencies and Disasters, v.8, n.3, p.301-324, nov. 1990. Disponível em: <http://ijmed.org/articles/21/download/>, Acesso em: 19 mar. 2014.

BURTON, I. et al. G. The Environment as Hazard. New York: Oxford University Press, 1978.

CAPRA, F. O ponto de mutação. $15^{\circ}$ ed. São Paulo: Cultrix, 1982.

CMMAD - COMISSÃO MUNDIAL SOBRE MEIO AMBIENTE E DESENVOLVIMENTO. Nosso futuro comum. Rio de Janeiro: Fundação Getulio Vargas, 1988.

CUTTER, S. L. The geography of social vulnerability: race, class, and catastrophe. In: Understanding Katrina: Perspectives from the Social Sciences. 2006. Disponível em: <http://understandingkatrina.ssrc.org/Cutter/>. Acesso em: 24 jul. 2016.

DE LONG, D.; DAVENPORT, T.; BEERS, M. Research note: What is a knowledge management project? 1997. Disponível em: <http://www.providersedge.com/docs/ km_articles/what_is_a_km_project.pdf>. Acesso em: $1^{\circ}$ mai. 2016.

FUNTOWICZ, S. O.; DE MARCHI, B. Ciência pós-normal, complexidade reflexiva e sustentabilidade. In: LEFF, E. (Coord.) A complexidade ambiental. Trad. Eliete Wolff. São Paulo: Cortez, 2003. p.65-98.

FUNTOWICZ, S. O.; RAVETZ, J. R. Ciência pós-normal e comunidades ampliadas de pares face aos desafios ambientais. História, Ciências, Saúde, Rio de Janeiro, v.IV, n.2, p.219-30, jul.-out, 1997. Disponível em: <http://www.scielo.br/pdf/hcsm/v4n2/ v4n2a01.pdf>. Acesso em: 10 abr. 2011.

. La ciencia posnormal: la ciencia con la gente. Barcelona: Icaria, 2000.

FURTADO, C. O mito do desenvolvimento econômico. São Paulo: Paz e Terra, 1974.

GADOTTI, M. Educar para a sustentabilidade: uma contribuição à década da educação para o desenvolvimento sustentável. São Paulo: Editora Paulo Freire, 2008.

GIDDENS, A.; BECK, U.; LASH, S. Modernización reflexiva: política, tradición y estética en el orden social moderno. s. 1.: Alianza Editorial, 1997. 
HEWITT, K. (Ed.) Interpretations of Calamity. Boston: Mass Allen \& Unwin, 1983.

JONES, D. Environmental hazards in the 1990s: problems, paradigms and prospects. Geography, v.78, n.2, p.161-5, 1993.

JUNEJA, S. Disasters and Poverty: The risk nexus - A review of literature. Background. Paper for the 2009 ISDR Global Assessment Report on Disaster Risk Reduction. UNISDR, Geneva, august, 2009.

KEEN, M.; BROWN, V. A.; DYBALL, R. Social learning: a new approach to environmental management. In: Social Learning in environmental management: Towards a Sustainable Future. London: Earthscan, 2005. p.3-21.

KILVINGTON, M. J. Social Learning as a framework for building capacity to work, nov. 2007. Disponível em: <http://www.landcareresearch.co.nz/publications/researchpubs/Social_learning_review.pdf>, Acesso em: $1^{\circ}$ maio 2016.

Building capacity for social learning in environmental management. 2010. These (Ph.D Philosophy) - Lincoln University. Canterbury, Nova Zelândia, 2010. Disponível em: <http://goo.gl/Zde6Cc>, Acesso em: 28 jan. 2013.

KUHN, T. S. A estrutura das revoluções cientificas. $9^{\circ}$ ed. São Paulo: Perspectiva, 2005. LEFF, E. Pensar a complexidade ambiental. In: (Coord.) A complexidade ambiental. Trad. Eliete Wolff. São Paulo: Cortez, 2003.

Racionalidade ambiental: a reapropriação social da natureza. Rio de Janeiro: Civilização Brasileira, 2006.

LIDSTONE, J. Disaster education in the school curriculum. In: INGLETON, J. (Ed.) Natural disaster management. Leicester: Tudor Rose, 1999. p.235-45.

LIMA, G. F. C. O discurso da sustentabilidade e suas implicações para a educação. Ambiente e Sociedade, Campinas, v.6, n.2, jul./dez. 2003.

MILETI, D. S. Disasters by design. In: BRITTON, N. R. (Ed.) The changing risk landscape: implications for insurance risk management. Aon Group Australia Limited Conference, 1999. Disponível em: <http://www.aonlineaon.com/public/intelligence/disasters_by_design.pdf>, Acesso em: 24 out. 2013.

NORGAARD, R. B. Development betrayed: the end of the progress and a coevolutionary revisioning of the future. Estados Unidos; Canadá: Routledge, 1994.

PETAL, M. Disaster prevention for schools: guidance for education sector decisionmakers. Suíça, UNISDR - Thematic Platform for Knowledge and Education, 2008. Disponível em: <http://www.preventionweb.net/english/professional/trainings-events/edumaterials/v.php?id=7344>. Acesso em: 24 mar. 2014.

PETAL, M.; GREEN, R.; KELMAN, I.; SHAW, R.; DIXIT, A. Community-based construction for disaster risk reduction. In: BOSHER, L. Hazards and the built environment: attaining built-in resilience. s. 1.: Taylor \& Francis; Routledge, 2008. p.191217. Disponível em: <http://goo.gl/H7PhfK>, Acesso em: 20 jun. 2014.

RAVETZ, J. R. Post-normal science and the complexity of transitions towards sustainability. Ecological Complexity, 3, p.275-84, 2006.

RAVETZ, J. R.; DE MARCHI, B. Risk management and governance: a post-normal Science approach. Futures, n.31, p.743-57, 1999. 
ROE, E. Narrative policy analysis: theory and practice. Durham, CT: Duke University Press, 1994.

SILVA, F. C.; MACEDO, E. S. Percepção ambiental e riscos naturais com enfoque em deslizamentos. In: SIMPÓSIO BRASILEIRO DE DESASTRES NATURAIS E TECNOLÓGICOS - SIBRADEN, II. Santos, Anais..., dez, 2007.

SMITH K.; PETLEY, D. N. Environmental hazards: assessing risk and reducing disaster. $5^{\circ}$ ed. London; New York: Routledge, 2009.

SULAIMAN, S. N. De que adianta? O papel da educação para prevenção de desastres naturais. São Paulo, 2014. Tese (Doutorado em Educação) - Faculdade de Educação, Universidade de São Paulo. São Paulo, 2014.

TIMMERMAN, P. Vulnerability, resilience and the collapse of society: a review of models and possible climatic applications. Toronto: University of Toronto, 1981.

UNESCO - Organização das Nações Unidas para a Educação, a Ciência e a Cultura. Década das Nações Unidas da educação para o desenvolvimento sustentável (2005-2014). Documento final - Plano Internacional de Implementação. Brasília, Unesco no Brasil, 2005. Disponível em: <http://unesdoc.unesco.org/images/0013/001399/139937por. pdf>, Acesso em: 19 nov. 2012.

WARNER, J.; WAALEWIJN, P.; HILHORST, D. Public participation in Disaster-Prone Watersheds: Time for Multi-Stakeholder Platforms? Disaster site 6, Wageningen University. Irrigation and water management group. Disaster studies, 2002.

WESSELINK, A.; HOPPE, R. If post normal science is the solution, what is the problem?: The politics of activist environmental science. Science, Technology \& Human Values, v.36, n.3, p.389-412, 2013.

WISNER, B.; P. BLAIKIE; T. CANNON; I. DAVID. At risk: natural hazards, people's vulnerability and disasters. 2 ed. London; New York: Routledge, 2004.

RESUMO - Estudos sobre riscos de desastres naturais têm-se aprimorado de uma abordagem fisicalista para uma perspectiva socioambiental. No entanto, planejamento e gestão ainda seguem o paradigma antropocêntrico da superioridade humana e do poder ilimitado da ciência e tecnologia. Evidencia-se uma incapacidade cognitiva, cultural e de ação por parte de especialistas, científicos e tomadores de decisão (claimmakers) para identificar e atuar sobre as causas sociais da produção de risco. Frente a uma ciência cartesiana e positivista na resolução de problemas, baseada na segurança e controle sobre o mundo natural, propõe-se uma ciência pós-normal que considera os riscos e incertezas do conhecimento científico e das problemáticas ambientais. Essa nova proposta também incide sobre a participação e o diálogo entre stakeholders como referência para ampliar a qualidade do saber científico e o entendimento da complexidade das questões ambientais. Este artigo discute a necessidade de se promover um salto epistemológico sobre a forma de pensar e produzir conhecimentos, bem como implementar a gestão dos riscos de desastres, tendo como objeto de estudo processos de comunicação e educação para prevenção de desastres.

PALAVRAS-CHAVE: Desastres naturais, Inquestionabilidade do risco, Ciência pós-normal, Educação. 
ABSTRACT - Studies on the risks of natural disasters have improved from a physicalist approach to a social and environmental perspective. However, planning and management still follow the anthropocentric paradigm of human superiority and the unlimited power of science and technology, evincing a cognitive, cultural and action inability on the part of experts, scientists and decision makers (or, rather, claim makers) to identify and act upon the social causes of risk production. In view of the Cartesian and Positivist science used to solve problems, based on security and on control over the natural world, a post-normal science has been proposed that considers the risks and uncertainties of scientific knowledge and environmental issues. This new approach encompasses participation and dialogue among stakeholders as a means to increase the quality of scientific knowledge and acknowledge the complexity of environmental issues. This article discusses the need for an epistemological leap on how we think and produce knowledge, as well as for implementing the management of disaster risk. Its objects of study are communication processes and education for disaster prevention.

KEYWORDS: Natural disasters, Unquestionability of risk, Post-normal science, Education.

Samia Nascimento Sulaiman é doutora em Educação pela Universidade de São Paulo, e doutora em Gestão integral da Água, pela Universidad de Alicante, Espanha.

@ - samia.sulaiman@gmail.com

Antonio Aledo é professor titular do Departamento de Sociologia I, Universidad de Alicante, Espanha. @- antonio.aledo@ua.es

Recebido em 31.8.2016 e aceito em 23.9.2016.

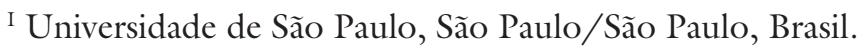

II Departamento de Sociologia I, Universidad de Alicante, Alicante, Espanha. 